\title{
Prevalence of Internet Addiction and Gaming Disorders in Southeast Asia: A Meta-Analysis
}

\author{
Doris X. Y. Chia ${ }^{1}$, Charis W. L. Ng ${ }^{1}{ }^{10}$, Gomathinayagam Kandasami ${ }^{1}$, Mavis Y. L. Seow ${ }^{2,3}$, \\ Carol C. Choo $\left.{ }^{4} \mathbb{D}^{\text {, Peter K. H. Chew }}{ }^{5}{ }^{(}\right)$, Cheng Lee ${ }^{1(\mathbb{D}}$ and Melvyn W. B. Zhang ${ }^{1,6, *}$
}

1 National Addictions Management Service, Institute of Mental Health, Singapore 539747, Singapore; doris_xy_chia@imh.com.sg (D.X.Y.C.); charis_wl_ng@imh.com.sg (C.W.L.N.); gomathinayagam_kandasami@imh.com.sg (G.K.); cheng_lee@imh.com.sg (C.L.) Department of Psychology, Institute of Mental Health, Singapore 539747, Singapore; mavis_seow@imh.com.sg

3 Department of Psychology, James Cook University, Singapore 387380, Singapore

4 Te Wānanga o Ngā Kete, Department of Arts, Law, Psychology \& Social Sciences, University of Waikato, Hamilton 3240, New Zealand; ccychoo@gmail.com

5 College of Healthcare Sciences, Division of Tropical Health and Medicine, James Cook University, Townsville QLD 4811, Australia; peter.chew@jcu.edu.au

6 Family Medicine \& Primary Care, Lee Kong Chian School of Medicine, Nanyang Technological University Singapore, Singapore 308232, Singapore

* Correspondence: melvynzhangweibin@gmail.com; Tel.: +65-3892504

Received: 16 March 2020; Accepted: 8 April 2020; Published: 9 April 2020

\begin{abstract}
This meta-analytic review aimed to examine the pooled prevalence rates of Internet addiction and gaming disorders in Southeast Asia. Several databases including PubMed, MEDLINE, PsycINFO, Web of Science, Embase, and Cochrane Central were searched and a total of 24 studies were included in this study. The selection of studies was conducted in accordance to the preferred reporting items for systematic reviews and meta-analyses (PRISMA) guidelines. Two meta-analyses were conducted to examine data on Internet addiction and gaming disorders separately. A random-effects model was employed to derive the pooled prevalence rate. Mixed-effects meta-regression and subgroup analyses were performed to examine the moderators of the between-study heterogeneity. Publication bias was tested using the Egger's regression test and funnel plot. Only seven out of the 11 Southeast Asian countries were represented in the literature. All except for two of the included studies were cross-sectional in nature. The findings revealed a pooled prevalence rate of $20.0 \%(95 \%$ confidence interval: $14.5 \%-27.0 \%$ ) and $10.1 \%$ (95\% confidence interval: $7.3 \%-13.8 \%$ ) for Internet addiction and gaming disorders respectively. Mean age and study population were significant moderators of the between-study heterogeneity in the prevalence rates of gaming disorders such that samples involving older participants showed higher prevalence rate than those involving younger individuals. Country of study was found to be significant moderator of the between-heterogeneity for both Internet addiction and gaming disorders, however the findings should be interpreted with caution due to the small and unbalanced sample sizes. There was no significant publication bias. Such epidemiology research should be extended to the Southeast Asian countries that have not been studied or are under-studied. Given that the prevalence rates appear to be higher in Southeast Asia than in other world regions, future research should also explore the factors behind these inter-regional differences. Further longitudinal studies should also be conducted to examine the trajectories of such disorders.
\end{abstract}

Keywords: Internet addiction; gaming disorders; Southeast Asia; prevalence; meta-analysis 


\section{Introduction}

Over the past few decades, technological breakthroughs have fundamentally shaped the role of the Internet in our lives. The Internet has now become an integral part of our daily living across school, work, and leisure. In Southeast Asia, the Internet Penetration Rate (IPR), defined as the percentage of the total population who use the Internet, was the highest for Brunei as of June 2019 (94.9\%), followed by Singapore $(84.5 \%)$ and Thailand $(82.2 \%)$ [1].

This widespread use of the Internet has resulted in significant concerns with regards to problematic Internet behaviors and related conditions. Based on the Diagnostic and Statistical Manual of Mental Disorders, 4th Edition (DSM-IV) diagnostic criteria on Pathological Gambling, Young [2] has defined Internet addiction as the excessive use of Internet for leisure purposes over six months meeting five or more of the following criteria: preoccupation with the Internet; spending longer time online to achieve gratification; unsuccessful attempts to reduce or quit Internet use; experience of negative moods such as irritability associated with cut-down or quit attempts; using the Internet for longer periods than expected; negatively impacted school, work, or relationships; concealment about the extent of Internet use; and using the Internet to cope with problems or negative moods. Similarly, the American Psychiatric Association [3] has suggested for the inclusion of Internet Gaming Disorder (IGD) as a condition for further study in the latest edition of the Diagnostic and Statistical Manual of Mental Disorders (DSM-5). The DSM-5 outlines IGD as the presence of dysfunctional online gaming behavior that is marked by at least five of the proposed symptoms: preoccupation, psychological withdrawal, tolerance, failure to cut down or stop, loss of interest in previously enjoyable pursuits, constant gaming despite ramifications, lying about time spent on gaming, using gaming to cope with negative affect, and possible or actual negative repercussions on employment and social relationships due to gaming [3]. Whilst there have been suggested diagnostic criteria for both Internet addiction and gaming disorders, thus far, only gaming disorders have been officially recognized as a psychiatric disorder. The World Health Organization [4] has also formally included Gaming Disorder (GD) as a mental health condition in the recently updated International Classification of Diseases (ICD-11). The ICD-11 recognizes GD as dysfunctional gaming behavior, both online and offline, marked by inability to control, prioritization of gaming over other activities, and persistent or exacerbated gaming behavior despite negative life consequences [4].

Whilst there have been developments in the refinement of the diagnostic criteria for these disorders, there has since been more research done in this area. Notably, there has been a proliferation of epidemiological studies done to establish the prevalence rates of Internet and gaming disorders globally [5,6]. Given the high IPR in Southeast Asia, it is of vast importance to synthesize independent studies to achieve a holistic understanding on the prevalence rates of such disorders in the region. These rates will help facilitate policy planning and the allocation of resources both for the screening and the treatment of such disorders in the region.

To the authors' best of knowledge, there has only been one such study, Balhara and colleagues [7], that has attempted to conduct a narrative review in this area. However, whilst the aim of their review was to focus on Southeast Asian studies, only two out of the 29 epidemiological studies examined were conducted in the Southeast Asia region (Indonesia and Thailand). The majority of the included studies (22 out of 29) were conducted in India, which is a South Asian country. The Southeast Asian countries include Brunei, Myanmar, Cambodia, Timor-Leste, Indonesia, Laos, Malaysia, Philippines, Singapore, Thailand, and Vietnam. Hence, the prior review has not provided an accurate review of the rates of these disorders in Southeast Asia. In addition, the review was centered solely on studies conducted on student samples.

In order to address this research gap, this current study aimed to examine the pooled prevalence rates of Internet addiction and gaming disorders in Southeast Asia through a meta-analytic review of the available epidemiological studies conducted in the region. Meta-analysis is a quantitative approach to literature synthesis through the use of statistics and significance testing [8,9]. As compared to traditional qualitative methods, such as narrative and systematic reviews which are prone to 
subjectivity due to human interpretation, meta-analysis allows for a more objective literature synthesis by quantifying outcomes $[8,9]$. This is especially useful for the synthesis of epidemiological studies, such as the current study, where a pooled estimate of the prevalence rates can be calculated. In addition, moderators driving the between-study heterogeneity can also be explored using the heterogeneity indices [10]. Past epidemiological studies on Internet and gaming disorders have primarily focused on single age group, with a lack of comparison across groups. As such, this study aimed to examine the moderating effect of age group, operationalized as population type (e.g., children, adolescents, adults, or mixed) and mean age, on the between-study heterogeneity. This study also examines gender as a moderator, as past studies have shown that males are more susceptible to such disorders [11,12]. Lastly, inter-regional differences were also explored using country as a moderator. As compared to Balhara et al. [7], the current study has a broader focus by taking into account studies conducted on general populations, inclusive of non-student samples.

\section{Methods}

\subsection{Search Strategy}

In order to identify the relevant articles, the following databases were searched from inception till 26th January 2020, that of PubMed, MEDLINE, PsycINFO, Web of Science, Embase, and Cochrane Central. The following search terminologies were used for MEDLINE, that of "epidemiology" or "prevalence" and "Internet" or "gaming" or "digital gaming" and "addict" or "disorder" or "problematic" or "pathological" or "excessive" or "dependence" or "addictive behaviour". As the interest was on articles from Southeast Asian countries, the following additional search terminologies were applied, that of "Thai" or "Singapore" or "Malaysia" or "Indonesia" or "Brunei" or "Myanmar" or "Vietnam" or "Cambodia" or "Lao" or "Filipino" or "Philippines" or "Southeast Asia". This MEDLINE search strategy was adapted for the rest of the databases. The search strategy for each of the databases have been included as a Supplementary Materials file. The authors have consulted a library information specialist in the conceptualization of the terminologies and key words used in the search strategy, and in the refinement of the search for the various databases.

\subsection{Inclusion and Exclusion Criteria}

Studies were included if they were: (1) original research, (2) examined prevalence rates of Internet or gaming disorders, (3) conducted in the Southeast Asia region, (4) conducted in general populations, and (5) the abstract was available in English language. Studies were excluded if they were: (1) non-original research (such as review studies), (2) did not report prevalence rates of Internet or gaming disorders, (3) not conducted within the Southeast Asia region, (4) conducted with psychiatric populations, or (5) the abstract was not available in English language.

\subsection{Ethical Approval}

As meta-analysis is considered to be a form of secondary research involving the extraction of findings from prior studies, therefore ethical approval was not required for the current study.

\subsection{Selection of Studies}

The selection of studies was conducted in accordance to the preferred reporting items for systematic reviews and meta-analyses (PRISMA) guidelines. The title and abstract of the studies identified from the databases using the aforementioned search strategy were first screened for relevancy to the study topic on Internet and gaming disorders by two independent authors. The full-text article of the remaining studies were then assessed against the inclusion and exclusion criteria to determine the final pool of studies to be included in the current meta-analysis. 


\subsection{Data Extraction}

Data from the included studies on Internet addiction and gaming disorders were extracted separately into two standardized spreadsheets by author DC. The following data were extracted: (1) citation including last name of first author and year of publication, (2) country in which the study was conducted, (3) study design, (4) study population, (5) sample size, (6) characteristics of sample including gender breakdown and mean age, (7) assessment tool utilized, and (8) prevalence rate. To ensure data accuracy, the extracted data were cross-checked by a second author (MZ). Any disagreements were resolved by means of a discussion between the authors DC and MZ. If they were still not able to resolve the disagreements, a third author was consulted.

\subsection{Data Analyses}

All statistical analyses were conducted using the Comprehensive Meta-Analysis Version 2.0 Program. The extracted data on Internet addiction and gaming disorders were analyzed separately via two independent meta-analyses. For the purpose of meta-analysis, the extracted data were coded into formats that were compatible with the program, e.g., categorical variables in text format were coded into numerical categories and percentages were represented in decimal format.

For each of the two conditions, a random-effects model was employed to derive the pooled estimate of the prevalence rates, based on $95 \%$ confidence interval. This type of model assumes that the included studies are a subset of all possible relevant studies, accounting for true heterogeneity in effect sizes across studies on top of systematic sampling error, allowing for the generalization of findings beyond the context of the included studies $[10,13]$. A forest plot was also generated to provide a visual representation of the prevalence data from the included studies.

Heterogeneity across studies was assessed through significance testing of the $\mathrm{I}^{2}$ statistic, with null hypothesis assuming homogeneity. Based on the heuristic recommended in Higgins et al. [14], an $\mathrm{I}^{2}$ of $25 \%, 50 \%$, and $75 \%$ reflect low, moderate, and high levels of heterogeneity respectively.

Following the significant between-studies heterogeneity found, mixed-effects meta-regression analyses using unrestricted maximum likelihood estimation were conducted to ascertain if the continuous moderators, including mean age and proportion of male participants, were significantly associated with the between-study heterogeneity in prevalence rates. For meta-regression, the regression coefficients and the corresponding $Z$ and $p$ values were reported. For categorical moderators, including country and study population, sub-group analyses using a mixed-effects model were done.

A funnel plot was generated to assess publication bias, with asymmetry suggesting possible presence of publication bias. Egger's regression test was also conducted to examine the significance of publication bias, with $p<0.05$ signifying the presence of a significant publication bias. If a significant publication bias was shown, a Classic Fail-Safe test would be conducted to ascertain the number of omitted studies needed to counter the publication bias.

\section{Results}

An initial pool of 184 citations were identified from the databases based on the search strategy. A total of 27 articles were found to be duplicates and were excluded. The screening of study titles and abstracts for relevancy to study topic resulted in the exclusion of 110 citations. Another 23 studies were subsequently excluded after the assessment of full-text article against the inclusion and exclusion criteria: did not report prevalence data on Internet addiction or gaming disorders (12 studies), non-original study (one study), use of psychiatric sample (one study), no access to full-text article (six studies), and sharing of duplicated dataset with an included study (three studies). A final count of 24 studies were included for the current study (refer to Figure 1). The aforementioned steps on the selection of studies are detailed in Figure 1. 
184 citations were found using the search strategy.

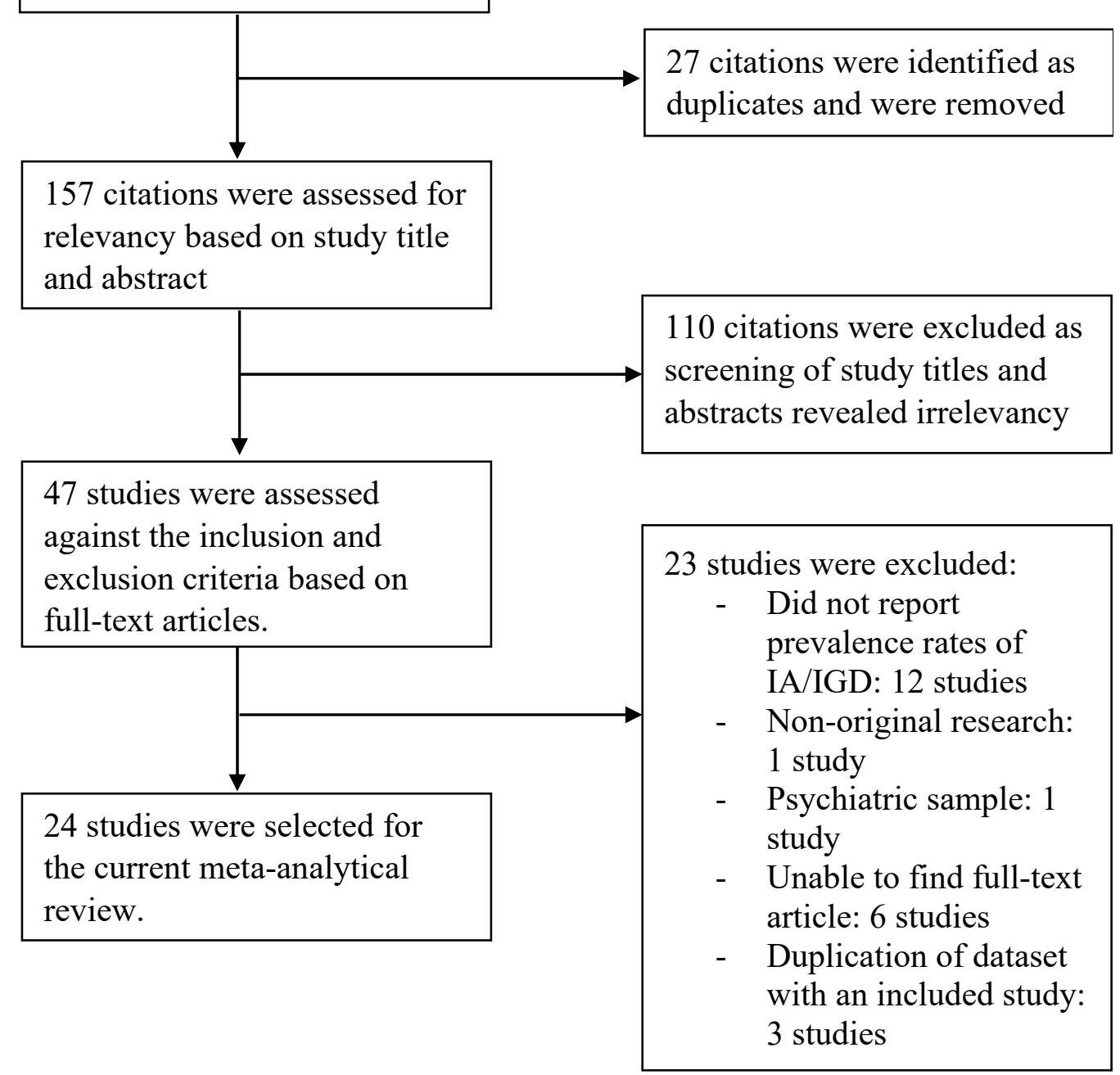

Figure 1. Flowchart depicting the process of study selection.

Of the included studies, eight studies were conducted in Malaysia, six in Singapore, five in Thailand, three in Vietnam, one study examined data from Malaysia and Philippines, and the remaining study looked at data from Indonesia, Malaysia, Myanmar, Thailand, and Vietnam. In terms of the condition studied, 16 studies were conducted on Internet addiction, six studies on Internet gaming disorder or gaming disorders, and two studies looked at both Internet addiction and gaming disorders. The study populations included adults (12 studies), adolescents (six studies), children (one study), children and adolescents (two studies), and adolescents and adults (three studies). All except for two studies were cross-sectional in nature.

For studies that examined more than one condition or country, the data on each condition or country were considered as unique data points for analysis. The characteristics of the included studies on Internet addiction and gaming disorders, including study details, country, study design, population type, sample size, mean age, gender breakdown, assessment tool, and prevalence rates, can be found in Tables 1 and 2, respectively. 
Table 1. Characteristics of the included studies on Internet addiction.

\begin{tabular}{|c|c|c|c|c|c|c|c|}
\hline Paper & Country & Study Design & Population & Sample Size & Sample Characteristics & Assessment Tool & Prevalence \\
\hline $\begin{array}{c}\text { Ainin et al. (2017) } \\
\text { [15] }\end{array}$ & Malaysia & Cross-sectional & Adults & 1000 & $\begin{array}{c}50.3 \% \text { males and } 49.7 \% \text { females } \\
\text { Mean age = N.A. years old (SD = N.A.) }\end{array}$ & $\begin{array}{l}\text { 20-item Young's Internet } \\
\text { Addiction Test (IAT) }\end{array}$ & $13.3 \%$ \\
\hline $\begin{array}{l}\text { Azmi et al. (2019) } \\
\text { [16] }\end{array}$ & Malaysia & Cross-sectional & Adolescents & 178 & $\begin{array}{c}36.5 \% \text { males and } 63.5 \% \text { females } \\
\text { Mean age }=10.6 \text { years old }(S D=1.57)\end{array}$ & $\begin{array}{l}\text { Malay Validated } \\
\text { Internet Addiction Test } \\
\text { (MVIAT) }\end{array}$ & $23.0 \%$ \\
\hline $\begin{array}{l}\text { Boonvisudhi et al. } \\
\text { (2017) [17] }\end{array}$ & Thailand & Cross-sectional & Adults & 705 & $\begin{array}{c}\text { N.A. } \% \text { males and N.A. } \% \text { females } \\
\text { Mean age }=20.51 \text { years old }(S D=1.91)\end{array}$ & $\begin{array}{l}\text { Young Diagnostic } \\
\text { Questionnaire (YDQ) }\end{array}$ & $24.4 \%$ \\
\hline $\begin{array}{l}\text { Ching et al. (2017) } \\
\text { [11] }\end{array}$ & Malaysia & Cross-sectional & Adults & 426 & $\begin{array}{c}36.6 \% \text { males and } 63.4 \% \text { females } \\
\text { Mean age = 21.6 years old }(\mathrm{SD}=1.5)\end{array}$ & MVIAT & $36.9 \%$ \\
\hline Ke et al. (2018) [18] & Malaysia & Longitudinal & Adolescents & 157 & $\begin{array}{c}46.0 \% \text { males and } 54.0 \% \text { females } \\
\text { Mean age = } 14.0 \text { years old (SD = N.A.) }\end{array}$ & $\begin{array}{l}\text { Problematic Internet } \\
\text { Use Questionnaire } \\
\text { (PIUQ) }\end{array}$ & $\begin{array}{l}92.4 \% \\
\text { (Time 1) }\end{array}$ \\
\hline $\begin{array}{l}\text { Mak et al. (2014a) } \\
\text { [19] }\end{array}$ & Malaysia & Cross-sectional & Adolescents & 969 & $\begin{array}{c}46.0 \% \text { males and } 54.0 \% \text { females } \\
\text { Mean age = } 14.7 \text { years old }(\mathrm{SD}=1.2)\end{array}$ & IAT & $2.4 \%$ \\
\hline $\begin{array}{l}\text { Mak et al. (2014b) } \\
\text { [19] }\end{array}$ & Philippines & Cross-sectional & Adolescents & 999 & $\begin{array}{c}38.5 \% \text { males and } 61.5 \% \text { females } \\
\text { Mean age }=16.4 \text { years old }(\mathrm{SD}=1.7)\end{array}$ & IAT & $4.9 \%$ \\
\hline $\begin{array}{l}\text { Ng et al. (2015) } \\
{[20]}\end{array}$ & Malaysia & Cross-sectional & Adults & 164 & $\begin{array}{c}34.1 \% \text { males and } 65.9 \% \text { females } \\
\text { Mean age }=19.02 \text { years old }(S D=0.19)\end{array}$ & YDQ & $43.9 \%$ \\
\hline $\begin{array}{l}\text { Norhizan et al. } \\
\text { (2019) [21] }\end{array}$ & Malaysia & Cross-sectional & Adults & 674 & $\begin{array}{c}23.0 \% \text { males and } 77.0 \% \text { females } \\
\text { Mean age }=21.66 \text { years old }(S D=1.89)\end{array}$ & IAT & $4.7 \%$ \\
\hline $\begin{array}{l}\text { Othman et al. } \\
\text { (2017) [22] }\end{array}$ & Malaysia & Cross-sectional & Adults & 267 & $\begin{array}{c}13.9 \% \text { males and } 86.1 \% \text { females } \\
\text { Mean age }=20.9 \text { years old }(S D=1.4)\end{array}$ & MVIAT & $1.1 \%$ \\
\hline $\begin{array}{l}\text { Simcharoen et al. } \\
\text { (2018) [23] }\end{array}$ & Thailand & Cross-sectional & Adults & 324 & $\begin{array}{c}43.2 \% \text { males and } 56.8 \% \text { females } \\
\text { Mean age }=20.88 \text { years old }(S D=1.81)\end{array}$ & Thai version of the IAT & $0.6 \%$ \\
\hline $\begin{array}{l}\text { Siraj et al. (2015) } \\
{[24]}\end{array}$ & Malaysia & Cross-sectional & Adults & 176 & $\begin{array}{c}26.7 \% \text { males and } 73.3 \% \text { females } \\
\text { Mean age = N.A. years old (SD = N.A.) }\end{array}$ & $\begin{array}{l}\text { Internet Addiction } \\
\text { Diagnostic } \\
\text { Questionnaire (IADQ) }\end{array}$ & $20.5 \%$ \\
\hline $\begin{array}{l}\text { Subramaniam et al. } \\
\text { (2008) [25] }\end{array}$ & Singapore & Cross-sectional & Adolescents & 2735 & $\begin{array}{c}49.3 \% \text { males and } 50.6 \% \text { females } \\
\text { Mean age }=13.9 \text { years old }(S D=1.0)\end{array}$ & $\begin{array}{l}>5 \mathrm{~h} \text { spent on the } \\
\text { Internet per day }\end{array}$ & $17.1 \%$ \\
\hline
\end{tabular}


Table 1. Cont.

\begin{tabular}{|c|c|c|c|c|c|c|c|}
\hline Paper & Country & Study Design & Population & Sample Size & Sample Characteristics & Assessment Tool & Prevalence \\
\hline $\begin{array}{c}\text { Tang et al. (2017a) } \\
{[26]}\end{array}$ & Singapore & Cross-sectional & Adults & 1107 & $\begin{array}{c}37.4 \% \text { males and } 62.6 \% \text { females } \\
\text { Mean age }=21.45 \text { years old }(\mathrm{SD}=1.80)\end{array}$ & 12-item Young's IAT & $4.9 \%$ \\
\hline $\begin{array}{l}\text { Tang et al. (2018a) } \\
{[27]}\end{array}$ & Singapore & Cross-sectional & Adults & 1119 & $\begin{array}{c}38.0 \% \text { males and } 61.9 \% \text { females } \\
\text { Mean age }=21.52 \text { years old }(S D=1.89)\end{array}$ & 12-item Young's IAT & $9.3 \%$ \\
\hline $\begin{array}{c}\text { Tran et al. (2017a) } \\
\text { [28] }\end{array}$ & Vietnam & Cross-sectional & $\begin{array}{l}\text { Adolescents and } \\
\text { Adults }\end{array}$ & 566 & $\begin{array}{c}38.9 \% \text { males and } 61.1 \% \text { females } \\
\text { Mean age = } 21.5 \text { years old }(\mathrm{SD}=3.8)\end{array}$ & $\begin{array}{l}\text { Vietnamese version of } \\
\text { the 12-item IAT }\end{array}$ & $21.2 \%$ \\
\hline $\begin{array}{c}\text { Tran et al. (2017b) } \\
\text { [29] }\end{array}$ & Vietnam & Cross-sectional & $\begin{array}{l}\text { Adolescents and } \\
\text { Adults }\end{array}$ & 589 & $\begin{array}{c}36.8 \% \text { males and } 63.2 \% \text { females } \\
\text { Mean age = 21.7 years old }(\mathrm{SD}=1.7)\end{array}$ & $\begin{array}{l}\text { Vietnamese version of } \\
\text { the 12-item IAT }\end{array}$ & $20.9 \%$ \\
\hline $\begin{array}{l}\text { Turnbull et al. } \\
\text { (2018a) [30] }\end{array}$ & Indonesia & Cross-sectional & Adults & 231 & $\begin{array}{c}\text { N.A. } \% \text { males and N.A. } \% \text { females } \\
\text { Mean age }=\text { N.A. years old }(S D=\text { N.A.) }\end{array}$ & YDQ & $38.5 \%$ \\
\hline $\begin{array}{l}\text { Turnbull et al. } \\
\text { (2018b) [30] }\end{array}$ & Malaysia & Cross-sectional & Adults & 1023 & $\begin{array}{c}\text { N.A. } \% \text { males and N.A. } \% \text { females } \\
\text { Mean age = N.A. years old (SD = N.A.) }\end{array}$ & YDQ & $28.9 \%$ \\
\hline $\begin{array}{l}\text { Turnbull et al. } \\
\text { (2018c) [30] }\end{array}$ & Myanmar & Cross-sectional & Adults & 386 & $\begin{array}{c}\text { N.A. } \% \text { males and N.A. } \% \text { females } \\
\text { Mean age }=\text { N.A. years old }(S D=\text { N.A. })\end{array}$ & YDQ & $16.1 \%$ \\
\hline $\begin{array}{l}\text { Turnbull et al. } \\
\text { (2018d) [30] }\end{array}$ & Thailand & Cross-sectional & Adults & 783 & $\begin{array}{c}\text { N.A. } \% \text { males and N.A. } \% \text { females } \\
\text { Mean age }=\text { N.A. years old }(S D=\text { N.A. })\end{array}$ & YDQ & $52.4 \%$ \\
\hline $\begin{array}{l}\text { Turnbull et al. } \\
\text { (2018e) [30] }\end{array}$ & Vietnam & Cross-sectional & Adults & 817 & $\begin{array}{c}\text { N.A. } \% \text { males and N.A. } \% \text { females } \\
\text { Mean age }=\text { N.A. years old (SD = N.A.) }\end{array}$ & YDQ & $37.5 \%$ \\
\hline $\begin{array}{l}\text { Balhara et al. } \\
\text { (2019) [31] }\end{array}$ & Vietnam & Cross-sectional & Adults & 617 & $\begin{array}{c}28.0 \% \text { males and } 72.0 \% \text { females } \\
\text { Mean age }=21.0 \text { years old }(\mathrm{SD}=2.3)\end{array}$ & $\begin{array}{c}\text { Generalized } \\
\text { Problematic Internet } \\
\text { Use Scale-2 (GPIUS2) }\end{array}$ & $11.7 \%$ \\
\hline
\end{tabular}


Table 2. Characteristics of the included studies for Internet Gaming Disorder/Gaming Disorder.

\begin{tabular}{|c|c|c|c|c|c|c|c|}
\hline Paper & Country & Study Design & Population & Sample Size & Sample Characteristics & Assessment Tool & Prevalence \\
\hline $\begin{array}{l}\text { Apisitwasana et al. } \\
\text { (2017) [32] }\end{array}$ & Thailand & Cross-sectional & Children & 295 & $\begin{array}{c}52.9 \% \text { males and } 47.1 \% \text { females } \\
\text { Mean age }=9.87 \text { years old }(\mathrm{SD}=0.7)\end{array}$ & $\begin{array}{l}\text { Game Addiction Screening Test } \\
\text { (GAST) }\end{array}$ & $7.5 \%$ \\
\hline $\begin{array}{l}\text { Choo et al. (2010) } \\
\text { [12] }\end{array}$ & Singapore & Cross-sectional & $\begin{array}{l}\text { Children and } \\
\text { Adolescents }\end{array}$ & 2998 & $\begin{array}{c}72.7 \% \text { males and } 27.3 \% \text { females } \\
\text { Mean age }=11.2 \text { years old }(S D=2.06)\end{array}$ & $\begin{array}{l}\text { 10-item screening tool based on } \\
\text { DSM-IV Pathological Gambling }\end{array}$ & $8.7 \%$ \\
\hline $\begin{array}{l}\text { Chupradit et al. } \\
\text { (2019) [33] }\end{array}$ & Thailand & Cross-sectional & Adolescents & 242 & $\begin{array}{c}33.5 \% \text { males and } 66.5 \% \text { females } \\
\text { Mean age }=\text { N.A. years old (SD = N.A.) }\end{array}$ & GAST & $5.8 \%$ \\
\hline $\begin{array}{l}\text { Gentile et al. } \\
\text { (2011) [34] }\end{array}$ & Singapore & Longitudinal & $\begin{array}{l}\text { Children and } \\
\text { Adolescents }\end{array}$ & 2998 & $\begin{array}{c}72.7 \% \text { males and } 27.3 \% \text { females } \\
\text { Mean age = N.A. years old (SD = N.A.) }\end{array}$ & $\begin{array}{l}\text { 10-item screening tool based on } \\
\text { DSM-IV Pathological Gambling }\end{array}$ & 9.9\% (Time 1) \\
\hline $\begin{array}{l}\text { Subramaniam et al. } \\
\text { (2016) [35] }\end{array}$ & Singapore & Cross-sectional & $\begin{array}{l}\text { Adolescents } \\
\text { and Adults }\end{array}$ & 972 & $\begin{array}{c}63.2 \% \text { males and } 36.8 \% \text { females } \\
\text { Mean age }=23.6 \text { years old }(\mathrm{SD}=5.0)\end{array}$ & $\begin{array}{l}\text { Internet Gaming Disorder } \\
\text { Questionnaire (IGDQ) }\end{array}$ & $17.7 \%$ \\
\hline $\begin{array}{l}\text { Taechoyotin et al. } \\
\text { (2020) [36] }\end{array}$ & Thailand & Cross-sectional & Adolescents & 5497 & $\begin{array}{c}48.1 \% \text { males and } 37.6 \% \text { females } \\
\text { Mean age }=\text { N.A. years old }(S D=\text { N.A.) }\end{array}$ & $\begin{array}{l}\text { Thai Version of the Internet gaming } \\
\text { disorder test (IGD-20 Test) }\end{array}$ & $5.4 \%$ \\
\hline $\begin{array}{l}\text { Tang et al. (2017b) } \\
{[26]}\end{array}$ & Singapore & Cross-sectional & Adults & 1107 & $\begin{array}{c}37.4 \% \text { males and } 62.6 \% \text { females } \\
\text { Mean age }=21.45 \text { years old }(\mathrm{SD}=1.80)\end{array}$ & $\begin{array}{l}\text { 12-item Problematic Online } \\
\text { Gaming Questionnaire }\end{array}$ & $15.4 \%$ \\
\hline $\begin{array}{l}\text { Tang et al. }(2018 b) \\
{[27]}\end{array}$ & Singapore & Cross-sectional & Adults & 1119 & $\begin{array}{c}38.0 \% \text { males and } 61.9 \% \text { females } \\
\text { Mean age }=21.52 \text { years old }(\mathrm{SD}=1.89)\end{array}$ & $\begin{array}{l}\text { 12-item Problematic Online } \\
\text { Gaming Questionnaire }\end{array}$ & $15.4 \%$ \\
\hline
\end{tabular}




\subsection{Internet Addiction}

From the meta-analysis based on a random-effects model, the pooled prevalence rate of Internet addiction was found to be $20.0 \%$ (95\% confidence interval: $14.5 \%-27.0 \%, Z=-6.955$, df $=22$, $\left.\tau^{2}=0.883, I^{2}=98.745\right)$. The forest plot shown in Figure 2 illustrates the prevalence rates found in the individual studies.

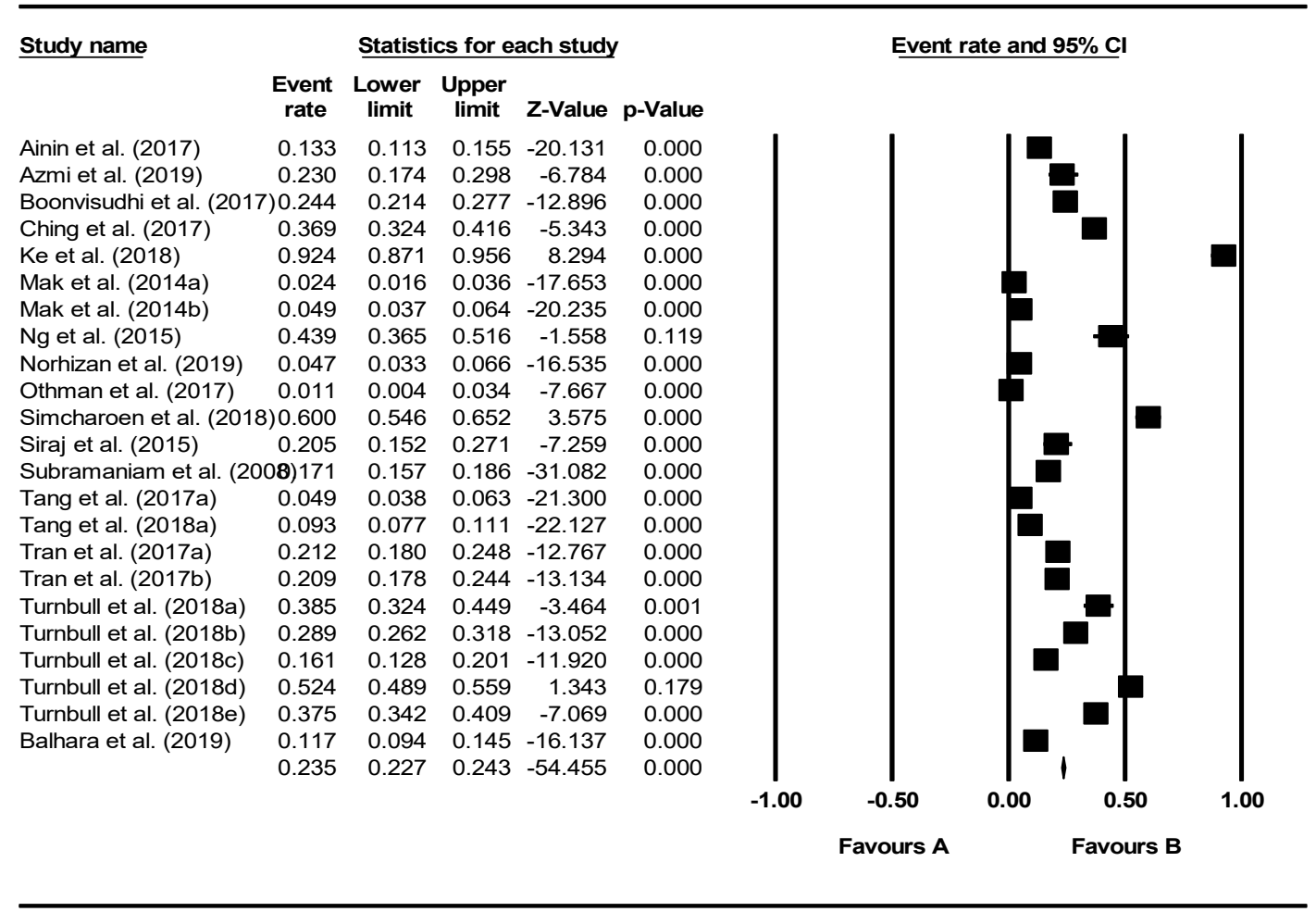

Figure 2. Forest plot of prevalence rates of Internet addiction.

There was significant between-study heterogeneity, $p<0.001$. Based on the heuristic recommended by Higgins et al. [14], the $I^{2}$ of $98.7 \%$ represented high level of heterogeneity. The mixed-effects meta-regression revealed that both mean age $(B=-0.084, Z=-0.746, p=0.456)$ and proportion of male participants $(B=7.024, Z=1.835, p=0.067)$ were not significant moderators of the between-study heterogeneity. The meta-regression findings are summarized in Table 3. The mixed-effects subgroup analyses showed that only country of study was a significant categorical moderator, with Thailand having the highest point estimate (44.7\%), followed by Indonesia (38.5\%), Vietnam (21.6\%), Malaysia $(19.2 \%)$, Myanmar (16.1\%), Singapore (9.4\%), and Philippines (4.9\%). On the other hand, study population was not found to be a significant moderator. The findings on the subgroup analyses can be found in Table 4.

Table 3. Meta-regression of mean age and proportion of male participants on prevalence rates of Internet addiction.

\begin{tabular}{cccccccc}
\hline Moderators & $\begin{array}{c}\text { Number } \\
\text { of Studies } \\
\text { Used }\end{array}$ & Slope & $\begin{array}{c}\text { Standard } \\
\text { Error }\end{array}$ & $\begin{array}{c}\text { 95\% CI: } \\
\text { Lower } \\
\text { Limit }\end{array}$ & $\begin{array}{c}\text { 95\% CI: } \\
\text { Upper } \\
\text { Limit }\end{array}$ & $\mathbf{Z}$ & $p$ \\
\hline $\begin{array}{c}\text { Mean age } \\
\text { Proportion of male }\end{array}$ & 16 & -0.084 & 0.112 & -0.303 & 0.136 & -0.746 & 0.456 \\
participants & 17 & 7.024 & 3.828 & -0.479 & 14.527 & 1.835 & 0.067 \\
\hline
\end{tabular}


Table 4. Subgroup analyses on the effect of country and population the prevalence rates of Internet addiction.

\begin{tabular}{ccccc}
\hline Subgroups & $\begin{array}{c}\text { No. of Studies } \\
\text { Used }\end{array}$ & $\begin{array}{c}\text { Pooled } \\
\text { Prevalence (\%) }\end{array}$ & $\mathbf{9 5 \% \text { CI }}$ & $\begin{array}{c}\boldsymbol{p} \text { Value for between-Group } \\
\text { Comparison }\end{array}$ \\
\hline Indonesia & 1 & 38.5 & $32.4-44.9$ & \\
Malaysia & 10 & 19.2 & $10.6-32.3$ & \\
Myanmar & 1 & 16.1 & $12.8-20.1$ & \\
Philippines & 1 & 4.9 & $3.7-6.4$ & \\
Singapore & 3 & 9.4 & $4.5-18.5$ & \\
Thailand & 3 & 44.7 & $24.8-66.5$ & \\
Vietnam & 4 & 21.6 & $12.8-34.1$ & \\
Overall: & 23 & 17.0 & $15-19.1$ & \\
Adults & 16 & 20.1 & $13.6-28.8$ & \\
Adolescents & 5 & 19.6 & $6.6-45.4$ & \\
Adolescents and & 2 & 21.0 & $18.8-23.5$ & \\
Adults & 23 & 21.0 & $18.8-23.3$ & 0.966 \\
Overall: & & &
\end{tabular}

CI: Confidence Interval. $p<0.001 *$.

There was no significant publication bias as indicated by the non-significant Egger' regression test (intercept $=-4.358,95 \%$ confidence interval $=-13.7 \%-5.0 \%, t=0.968, \mathrm{df}=21, p=0.344$ ).

\subsection{Gaming Disorders}

The pooled prevalence rate of gaming disorders based on a random-effects model was found to be $10.1 \%$ (95\% confidence interval: $\left.7.3 \%-13.8 \%, Z=-11.899, \mathrm{df}=7, \tau^{2}=0.253, I^{2}=97.4\right)$. The forest plot of prevalence rates of gaming disorders is illustrated in Figure 3.

\begin{tabular}{|c|c|c|c|c|c|c|c|c|c|c|}
\hline \multirow[t]{2}{*}{ Study name } & \multicolumn{5}{|c|}{ Statistics for each study } & \multicolumn{5}{|c|}{ Event rate and $95 \% \mathrm{Cl}$} \\
\hline & $\begin{array}{l}\text { Event } \\
\text { rate }\end{array}$ & $\begin{array}{l}\text { Lower } \\
\text { limit }\end{array}$ & $\begin{array}{l}\text { Upper } \\
\text { limit }\end{array}$ & Z-Value & $p$-Value & & & & & \\
\hline Apisitwasana et al. (2017) & 0.075 & 0.050 & 0.111 & -11.365 & 0.000 & & & & & \\
\hline Choo et al. (2010) & 0.087 & 0.077 & 0.098 & -36.277 & 0.000 & & & & & \\
\hline Chupradit et al. (2019) & 0.058 & 0.035 & 0.095 & -10.136 & 0.000 & & & & & \\
\hline Gentile et al. (2011) & 0.099 & 0.089 & 0.110 & -36.114 & 0.000 & & & & & \\
\hline Subramaniam et al. (2016) & 0.177 & 0.154 & 0.202 & -18.287 & 0.000 & & & & & \\
\hline Taechoyotin et al. (2020) & 0.054 & 0.048 & 0.060 & -47.981 & 0.000 & & & & & \\
\hline Tang et al. (2017b) & 0.154 & 0.134 & 0.176 & -20.459 & 0.000 & & & & & \\
\hline \multirow[t]{4}{*}{ Tang et al. (2018b) } & 0.154 & 0.134 & 0.176 & -20.569 & 0.000 & & & & & \\
\hline & 0.100 & 0.095 & 0.105 & -77.853 & 0.000 & & & & & \\
\hline & & & & & & -1.00 & -0.50 & 0.00 & 0.50 & 1.00 \\
\hline & & & & & & & Favours $A$ & & avours & \\
\hline
\end{tabular}

Figure 3. Forest plot of prevalence rates of gaming disorders.

There was significant between-study heterogeneity, $p<0.001$. According to the heuristic suggested by Higgins et al. [14], the $I^{2}$ of $97.4 \%$ represented a high level of heterogeneity. The mixed-effects meta-regression revealed that mean age $(B=0.065, Z=9.250, p=0.000)$ was a significant moderator of the between-study heterogeneity, while proportion of male participants was not significant $(B=0.027$, $Z=0.023, p=0.981$ ). The meta-regression findings are shown in Table 5 . The mixed-effects subgroup analyses showed that both the country of study $(B=0.069, Z=-34.074, p=0.000)$ and study population 
$(B=0.102, Z=-66.852, p=0.000)$ were significant categorical moderators. The findings on subgroup analyses are detailed in Table 6 . In particular, the studies conducted in Singapore showed higher pooled prevalence estimate of gaming disorders (13.0\%) than of Thailand (5.7\%). In terms of study population, the pooled prevalence estimate was the highest for adolescents and adults $(17.7 \%)$, followed by adults $(15.4 \%)$, children and adolescents $(9.3 \%)$, children $(7.5 \%)$, and adolescents $(5.4 \%)$.

Table 5. Meta-regression of mean age and proportion of male participants on prevalence rates of gaming disorders.

\begin{tabular}{cccccccc}
\hline Moderators & $\begin{array}{c}\text { Number } \\
\text { of Studies } \\
\text { Used }\end{array}$ & Slope & $\begin{array}{c}\text { Standard } \\
\text { Error }\end{array}$ & $\begin{array}{c}\text { 95\% CI: } \\
\text { Lower } \\
\text { Limit }\end{array}$ & $\begin{array}{c}\text { 95\% CI: } \\
\text { Upper } \\
\text { Limit }\end{array}$ & $\mathbf{Z}$ & $p$ \\
\hline Mean age & 5 & 0.065 & 0.007 & 0.051 & 0.079 & 9.250 & $0.000 *$ \\
\hline $\begin{array}{c}\text { Proportion of male } \\
\text { participants }\end{array}$ & 8 & 0.027 & 1.144 & -2.216 & 2.269 & 0.023 & 0.981 \\
\hline
\end{tabular}

CI: Confidence Interval. $p<0.001 *$.

Table 6. Subgroup analyses on the effect of country and population the prevalence rates of gaming disorders.

\begin{tabular}{ccccc}
\hline Subgroups & $\begin{array}{c}\text { No. of Studies } \\
\text { Used }\end{array}$ & $\begin{array}{c}\text { Pooled } \\
\text { Prevalence (\%) }\end{array}$ & $\mathbf{9 5 \% \text { CI }}$ & $\begin{array}{c}p \text { Value for between-Group } \\
\text { Comparison }\end{array}$ \\
\hline Singapore & 5 & 13.0 & $9.8-17.0$ & \\
Thailand & 3 & 5.7 & $4.8-6.7$ & $0.000^{*}$ \\
Overall: & 8 & 6.9 & $6.0-7.9$ & \\
Adults & 2 & 15.4 & $14.0-17.0$ & \\
Adolescents & 2 & 5.4 & $4.9-6.0$ & \\
Children & 1 & 7.5 & $5.0-11.1$ & \\
Children and Adolescents & 2 & 9.3 & $8.2-10.5$ & $0.000^{*}$ \\
Adolescents and Adults & 1 & 17.7 & $15.4-20.2$ & \\
Overall: & 8 & 10.2 & $9.6-10.8$ & \\
\hline
\end{tabular}

CI: Confidence Interval. $p<0.001 *$.

There was no significant publication bias as indicated by the non-significant Egger' regression test (intercept $=2.437,95 \%$ confidence interval $=-12.7 \%-17.6 \%, t=0.393, \mathrm{df}=6, p=0.708$ ).

\section{Discussion}

In terms of the IPR as of January 2020, the Southeast Asia region was ranked 9th (66.0\%), with the first being Northern Europe (95.0\%), followed by Western Europe (92.0\%), and Northern America $(88.0 \%)$ [37]. Despite the lower IPR, the pooled prevalence rates of Internet addiction $(20.0 \%)$ and gaming disorders $(10.1 \%)$ for the Southeast Asia region found in the current study are substantially higher than what have been found for other world regions. In their meta-analysis of 80 studies with mean participant age of 18.42 years old, Cheng et al. [38] found the global prevalence of Internet addiction to be $6.0 \%$ while the prevalence rates for the seven world regions examined were: Middle East (10.9\%), North America (8.0\%), Asia (7.1\%), South and East Europe (6.1\%), Oceania (4.3\%), North and West Europe (2.6\%), and South America (0.0\%). In terms of gaming, Müller et al. [39] examined the prevalence of Internet gaming disorder among seven European countries using data from 12,938 adolescents aged 14 to 17 and found the overall prevalence rate to be $1.6 \%$, with the highest rate found for Greece (2.5\%), followed by Poland (2.0\%), Iceland (1.8\%), Germany (1.6\%), Romania (1.3\%), The Netherlands (1.0\%), and Spain (0.6\%). Similarly, in their study of 1178 adolescents aged 8 to 18 in the United States, Gentile [40] found the prevalence rate of pathological videogaming to be $8.5 \%$. Cultural differences may offer a possible explanation for the higher prevalence rates observed in Southeast 
Asia despite the lower IPR. Western countries, such as the American and European countries, are often perceived as having a more individualistic culture than the Southeast Asian countries [41,42]. Interestingly, Arpaci et al. [43] found that a higher level of individualism, manifested by increased needs for achievement, autonomy, and dominance, and reduced need for affiliation, was significantly associated with a lower degree of Internet addiction. Accordingly, this higher level of individualism may have accounted for the lower prevalence of Internet and gaming disorders in Western countries than in Southeast Asia.

As indicated by the significant tests of heterogeneity and $I^{2}$ of $98.7 \%$ and $97.4 \%$, the current meta-analysis revealed high levels of between-study heterogeneity in the prevalence rates of Internet addiction and gaming disorders. The subsequent meta-regression and subgroup analyses explored mean age, proportion of male participants, country, and study population as potential moderators that may have contributed to the heterogeneity. In particular, mean age was found to be a significant moderator for gaming disorders, such that higher mean age of sample was associated with higher prevalence rate. Older individuals, such as college students and working adults, often have more autonomy and opportunities to utilize smart devices. This, coupled with their technological proficiency and the lack of external supervision, may put them at higher risk for gaming disorders. On the other hand, parents of younger children or adolescents, particularly in the Asian culture, may impose supervision and restrictions on their children's involvement in gaming behaviors due to academic concerns, reducing their risk for pathological gaming $[44,45]$. Besides the lack of external supervision, psychological motivations may also explain higher levels of gaming among older individuals. According to Erikson's Theory of Psychosocial Development [46], adolescents will actively explore and establish their self-identity at the "Identity versus Role Confusion" stage while adults will seek to build up their social relationships at the "Intimacy versus Isolation" stage. These intrapersonal and interpersonal needs are often shown to motivate gaming behaviors, particularly the involvement in the massively multiplayer online role-playing games [47,48]. In their study with 179 Korean undergraduates, Kim and Kim [47] found that social motivations significantly predicted excessive Internet gaming. In particular, the study showed that participants who were more motivated to overcome loneliness, build real-life social network, or increase virtual social network, were more likely to engage in excessive Internet gaming. In another study with 509 young adults in Croatia, it was revealed that lower levels of self-concept were associated with higher levels of problematic Internet gaming [48]. As such, adolescents and adults may be more inclined than children to play virtual games to build their self-identity and social relationships or to compensate such unfulfilled needs in real life. In a similar trend, the current study also revealed that study population was a significant moderator, such that samples from older populations including adolescents and adults $(17.7 \%)$ and adults only $(15.4 \%)$ showed higher prevalence rates of gaming disorders than samples from younger populations including children and adolescents (9.3\%), children (7.5\%), and adolescents (5.4\%). Interestingly, such trends were not observed for Internet addiction, such that both mean age and study population were not found to be significant moderators. This disparity in findings may be indicative of the distinctiveness and specificity of gaming disorders in the broader context of Internet addiction, which encompasses a wide range of other behaviors including gambling, social media, and shopping. However, these findings on the moderating effect of population type should be considered preliminary as the sample sizes of studies for each level of the moderator were small and unbalanced for Internet Addiction and gaming disorders respectively (adults $(n=16 ; n=2)$, adolescents $(n=5 ; n=2)$, children $(n=0$; $n=1)$, adolescents and adults $(n=2 ; n=1)$, and children and adolescents $(n=0 ; n=2))$. For both conditions, country of study was shown to be a significant moderator. Likewise, this finding should be interpreted with caution as not all Southeast Asian countries were represented, and for the represented countries, there was only limited and unbalanced number of studies. For instance, only seven out of the 11 Southeast Asian countries were represented in the epidemiological literature for Internet addiction (Malaysia $(n=10)$, Vietnam $(n=4)$, Singapore $(n=3)$, Thailand $(n=3)$, Indonesia $(n=1)$, 
Myanmar $(n=1)$, and Philippines $(n=1))$. Similarly, only two countries were represented for gaming disorders (Singapore $(n=5)$ and Thailand $(n=3)$ ).

Given the high levels of heterogeneity, there may be other moderators that were not explored in the current study due to the lack of data. For instance, the heterogeneity may be accounted for by the diversity in the assessment tools employed by the included studies i.e., varied assessment tools and varied cut-off criterions. A total of 10 different assessment tools were employed across the 23 studies on Internet addiction and five were used across the eight studies on gaming disorders. The varied assessment tools were observed to produce differing prevalence rates. Using the Internet Addiction Test (IAT) and the Revised Chen Internet Addiction Scale (CIAS-R), Mak et al. [19] examined the prevalence of Internet addiction in several countries, including Malaysia and Philippines, and found substantially different rates across the two scales. The study showed prevalence rates of $2.4 \%$ (IAT) and $14.1 \%$ (CIAS-R) for Malaysia, and 4.9\% (IAT) and 21.1\% (CIAS-R) for Philippines. Additionally, the use of varied cut-off criterions for the same scale across studies conducted on similar samples have also resulted in differing prevalence rates. In their studies to examine the prevalence of Internet addiction among college students in Singapore, Tang et al. $[26,27]$ employed the 12-item IAT with a cut-off of 40 points in one study and 36 points in the other to identify participants with Internet addiction. Despite the similarities in sampling, the two studies revealed differing rates of $4.9 \%$ and $9.3 \%$ respectively.

As with all studies, the current study has its own limitations. Firstly, despite having the aim of synthesizing the epidemiological studies on Internet and gaming disorders in Southeast Asia, not all Southeast Asian countries were represented due to the availability of literature. Moreover, the number of studies for each country was limited. The lack of studies may affect the generalizability of findings to Southeast Asia as a whole. Next, given the high levels of between-study heterogeneity, there may be other potential moderators that were not examined. As mentioned above, the assessment tools utilized in the included studies were diversified in terms of differing scales and cut-off criterions. However, these potential moderators were left unexplored as subgroup and meta-regression analyses were not feasible due to the limited number of studies. Additionally, the subgroup analyses on the moderating effects of population type and country on the between-study heterogeneity should be considered to be exploratory due to the small and unbalanced sample sizes of studies to represent each level of the moderators. In addition, the prevalence rate in one study, $\mathrm{Ng}$ et al. [20], did not correlate with the raw data provided in the table summarizing the characteristics of the participants, and another study (Boonvisudhi et al. [17]) did not report the gender breakdown based on the full sample which was included as a moderator in the current study. We have attempted to reach out to the authors via email for further clarifications, but to date, the authors have not responded. For the purpose of our current meta-analysis, we have recomputed the prevalence using the raw data results instead of the reported prevalence for $\mathrm{Ng}$ et al. [20] and excluded Boonvisudhi et al. [17] for the meta-regression which used proportion of male participants as the moderator. Lastly, given that MEDLINE and Scopus often have overlaps in journal indexing, only MEDLINE but not Scopus was searched for this current study. As such, there may be relevant studies not included in the current study as the journals in which they are published in are only indexed on Scopus. However, the authors note that such possibility is low due to the similarity in journal indexing across the two databases.

Despite the limitations, the current study is one of first efforts to synthesize the epidemiological literature on Internet and gaming disorders in the Southeast Asia context. Compared to traditional qualitative reviews, the study conducted meta-analysis which is a more robust and objective method to literature synthesis. By using meta-analysis, the study was able to derive pooled estimates as proxies of the prevalence of Internet and gaming disorders in Southeast Asia. Moreover, the study has also explored potential moderators that may influence the prevalence rates.

Through the current findings, several future research directions have been identified. To the best of the authors' knowledge, the epidemiology of Internet and gaming disorders have not been examined in some Southeast Asia countries including Brunei, Cambodia, Timor-Leste, and Laos. For those countries that have been covered, most were underrepresented. Accordingly, more research work 
can be done in the Southeast Asia countries to better understand such disorders in the region. Next, the study has revealed higher prevalence rates in Southeast Asia as compared to other world regions. Future studies should seek to explore the contributing factors and nuances behind these inter-regional differences. Lastly, all except for two of the included studies were cross-sectional in nature, future research may seek to conduct more longitudinal studies to examine the trajectories of Internet and gaming disorders.

There are several clinical implications that arise from the current findings. The prevalence of these disorders implies there being a need to screen for these disorders proactively amongst individuals who are coming forth for treatment. Screening should also be done amongst individuals with other psychiatric disorders, such as attention deficit hyperactivity disorder, social anxiety disorder, depressive disorder and alcohol abuse, as prior studies have reported there being an association between Internet Addiction, gaming disorders and these other psychiatric comorbidities [49,50].

\section{Conclusions}

In conclusion, the current meta-analysis found the pooled prevalence rates of Internet addiction and gaming disorders in Southeast Asia to be $20.0 \%$ and $10.1 \%$ respectively. The meta-regression and subgroup analyses showed that mean age and study population were significant moderators of the between-study heterogeneity in prevalence rates of gaming disorders with older samples showing higher prevalence rates than younger samples. Even though country of study was found to be a significant moderator for both Internet addiction and gaming disorders, findings should be interpreted with caution due to the small and unbalanced sample sizes. Future studies should extend such epidemiological research to Southeast Asian countries that have not been studied or under-studied. Given that the prevalence rates of Internet addiction and gaming disorders in Southeast Asia appear to be higher than that in other world regions, future research should examine the factors behind these inter-regional differences. Further longitudinal studies should also be conducted to examine the trajectories of such disorders.

Supplementary Materials: The following are available online at http:/www.mdpi.com/1660-4601/17/7/2582/s1, Supplementary Materials: Search Strategies.

Author Contributions: Conceptualization, P.K.H.C. and C.C.C.; Methodology, M.W.B.Z. and D.X.Y.C.; Formal Analysis, M.W.B.Z. and D.X.Y.C.; Data Curation, M.W.B.Z. and D.X.Y.C.; Writing-Original Draft Preparation, D.X.Y.C. and M.Y.L.S.; Writing-Review and Editing, all authors; Funding Acquisition, M.W.B.Z., C.W.L.N., D.X.Y.C., G.K., and C.L. All authors have read and agreed to the published version of the manuscript.

Funding: The publication costs were funded by the National Addictions Management Service, Institute of Mental Health, Singapore. The funding source was not involved in any part of this study.

Conflicts of Interest: The authors have no competing interests to declare.

\section{References}

1. Doan, E.Z. Internet penetration in Southeast Asia as of June 2019, by Country. Available online: https://www.statista.com/statistics/487965/internet-penetration-in-southeast-asian-countries/ (accessed on 13 December 2019).

2. Young, K.S. Internet addiction: A new clinical phenomenon and its consequences. Am. Behav. Sci. 2004, 48, 402-415. [CrossRef]

3. Parekh, R. Internet Gaming. Available online: https://www.psychiatry.org/patients-families/internet-gaming (accessed on 13 December 2019).

4. World Health Organization. Gaming Disorder. Available online: https://www.who.int/features/qa/gamingdisorder/en/ (accessed on 13 December 2019).

5. Kuss, D.J.; Griffiths, M.D.; Karila, L.; Billieux, J. Internet Addiction: A Systematic Review of Epidemiological Research for the Last Decade. Curr. Pharm. Des. 2014, 20, 4026-4052. [CrossRef] [PubMed]

6. Kuss, D.J.; Griffiths, M.D. Internet Gaming Addiction: A Systematic Review of Empirical Research. Int. J. Ment. Health Addict. 2012, 10, 278-296. [CrossRef] 
7. Balhara, Y.; Mahapatra, A.; Sharma, P.; Bhargava, R. Problematic internet use among students in South-East Asia: Current state of evidence. Indian J. Public Health 2018, 62, 197-210. [CrossRef]

8. Rosenthal, R.; DiMatteo, M.R. Meta-analysis: Recent Developments in Quantitative Methods for Literature Reviews. Annu. Rev. Psychol. 2001, 52, 59-82. [CrossRef] [PubMed]

9. Egger, M.; Smith, G.D.; Philips, A.N. Meta-analysis: Principles and procedures. BMJ 1997, 315, $1533-1537$. [CrossRef]

10. Zhang, M.W.B.; Ho, R.C.M.; Cheung, M.W.L.; Fu, E.; Mak, A. Prevalence of depressive symptoms in patients with chronic obstructive pulmonary disease: A systematic review, meta-analysis and meta-regression. Gen. Hosp. Psychiatry 2011, 33, 217-223. [CrossRef]

11. Ching, S.M.; Hamidin, A.; Vasudevan, R.; Sazlyna, M.; Wan Aliaa, W.; Foo, Y.L.; Yee, A.; Hoo, F. Prevalence and factors associated with internet addiction among medical students-A cross-sectional study in Malaysia. Med. J. Malays. 2017, 72, 7-11.

12. Choo, H.; Gentile, D.; Sim, T.; Li, D.D.; Khoo, A.; Liau, A. Pathological video-gaming among Singaporean youth. Ann. Acad. Med. 2010, 39, 822-829.

13. Borenstein, M.; Hedges, L.V.; Higgins, J.P.T.; Rothstein, H.R. A basic introduction to fixed-effect and random-effects models for meta-analysis. Res. Synth. Methods 2010, 1, 97-111. [CrossRef]

14. Higgins, J.P.T.; Thompson, S.G.; Deeks, J.J.; Altman, D.G. Measuring inconsistency in meta-analyses. BMJ 2003, 327, 557-560. [CrossRef] [PubMed]

15. Ainin, S.; Jaafar, N.I.; Ashraf, M.; Parveen, F. Exploring the role of demographics and psychological variables in internet addiction. Soc. Sci. Comput. Rev. 2017, 35, 770-780. [CrossRef]

16. Azmi, S.U.F.; Robson, N.; Othman, S.; Guan, N.C.; Isa, M.R. Prevalence and Risk Factors of Internet Addiction (IA) Among National Primary School Children in Malaysia. Int. J. Ment. Health Addict. 2019, 1-12. [CrossRef]

17. Boonvisudhi, T.; Kuladee, S. Association between Internet addiction and depression in Thai medical students at Faculty of Medicine, Ramathibodi Hospital. PLOS ONE 2017, 12. [CrossRef] [PubMed]

18. Ke, G.N.; Wong, S.F. Outcome of the Psychological Intervention Program: Internet Use for Youth. J. Ration. -Emot. Cogn. -Behav. Ther. 2019, 36, 187-200. [CrossRef]

19. Mak, K.-K.; Lai, C.-M.; Watanabe, H.; Kim, D.-I.; Bahar, N.; Ramos, M.; Young, K.S.; Ho, R.C.; Aum, N.-R.; Cheng, C. Epidemiology of internet behaviors and addiction among adolescents in six Asian countries. Cyberpsychol. Behav. Soc. Netw. 2014, 17, 720-728. [CrossRef]

20. Ng, C.G.; Isa, S.M.; Hashim, A.H.; Pillai, S.K.; Harbajan Singh, M.K. Validity of the Malay version of the Internet Addiction Test: A study on a group of medical students in Malaysia. Asia Pac. J. Public Health 2015, 27, NP2210-NP2219. [CrossRef]

21. Norhizan, N.F.A.; Ghazi, H.F.; Abdalrazak, H.A.; Abdalqader, M.A.; Baobaid, M.F.; Hasan, T.N.; Hassan, M.R. Social phobia and its association with body shape and internet addiction among private university students in Selangor, Malaysia. Int. J. Med Toxicol. Leg. Med. 2019, 22, 106-110. [CrossRef]

22. Othman, Z.; Lee, C.W.; Kueh, Y.C. Internet addiction and personality: Association with impulsive sensation seeking and neuroticism-anxiety traits. Int. Med. J 2017, 24, 375-378.

23. Simcharoen, S.; Pinyopornpanish, M.; Haoprom, P.; Kuntawong, P.; Wongpakaran, N.; Wongpakaran, T. Prevalence, associated factors and impact of loneliness and interpersonal problems on internet addiction: A study in Chiang Mai medical students. Asian J. Psychiatry 2018, 31, 2-7. [CrossRef]

24. Siraj, H.H.; Salam, A.; Hasan, N.A.B.; Jin, T.H.; Roslan, R.B.; Othman, M.N.B. Internet usage and academic performance: A study in a Malaysian public university. Int. Med. J. 2015, 22, 83-86.

25. Subramaniam, M.; Qiu, S.; Winslow, M. Prevalence and correlates of excessive Internet use among youth in Singapore. Ann. Acad. Med. Singap. 2008, 37, 9-14.

26. Tang, C.S.-K.; Koh, Y.W.; Gan, Y. Addiction to internet use, online gaming, and online social networking among young adults in China, Singapore, and the United States. Asia Pac. J. Public Health 2017, 29, 673-682. [CrossRef] [PubMed]

27. Tang, C.; Wu, A.; Yan, E.; Ko, J.; Kwon, J.; Yogo, M.; Gan, Y.; Koh, Y. Relative risks of Internet-related addictions and mood disturbances among college students: A 7-country/region comparison. Public Health 2018, 165, 16-25. [CrossRef] [PubMed] 
28. Tran, B.X.; Hinh, N.D.; Nguyen, L.H.; Le, B.N.; Nong, V.M.; Thuc, V.T.M.; Tho, T.D.; Latkin, C.; Zhang, M.W.; Ho, R.C. A study on the influence of internet addiction and online interpersonal influences on health-related quality of life in young Vietnamese. BMC Public Health 2017, 17, 138. [CrossRef]

29. Tran, B.X.; Mai, H.T.; Nguyen, L.H.; Nguyen, C.T.; Latkin, C.A.; Zhang, M.W.; Ho, R.C. Vietnamese validation of the short version of internet addiction test. Addict. Behav. Rep. 2017, 6, 45-50. [CrossRef]

30. Turnbull, N.; Peltzer, K.; Pengpid, S.; Low, W.Y.; Huu, T.N.; Rochmawati, E.; Win, H.H. Pathological Internet use and psychosocial risk factors among ASEAN university students. Iran. J. Psychiatry Behav. Sci. 2018, 12. [CrossRef]

31. Balhara, Y.P.S.; Doric, A.; Stevanovic, D.; Knez, R.; Singh, S.; Chowdhury, M.R.R.; Kafali, H.Y.; Sharma, P.; Vally, Z.; Vu, T.V. Correlates of Problematic Internet Use among college and university students in eight countries: An international cross-sectional study. Asian J. Psychiatry 2019, 45, 113-120. [CrossRef]

32. Apisitwasana, N.; Perngparn, U.; Cottler, L.B. Gaming Addiction Situation among Elementary School Students in Bangkok, Thailand. Indian J. Public Health Res. Dev. 2017, 8, 8-13. [CrossRef]

33. Chupradit, S.; Kaewmamuang, N.; Kienngam, N.; Chupradit, P.W. Prevalence and Correlates between Game Addiction and Stress of Adolescents in Chiang Mai, Thailand. Indian J. Public Health Res. Dev. 2019, 10, 1091-1096. [CrossRef]

34. Gentile, D.A.; Choo, H.; Liau, A.; Sim, T.; Li, D.; Fung, D.; Khoo, A. Pathological video game use among youths: A two-year longitudinal study. Pediatrics 2011, 127, e319-e329. [CrossRef] [PubMed]

35. Subramaniam, M.; Chua, B.Y.; Abdin, E.; Pang, S.; Satghare, P.; Vaingankar, J.A.; Chong, S. Prevalence and correlates of Internet gaming problem among Internet users: Results from an Internet survey. Ann. Acad. Med. Singap. 2016, 45, 174-183. [PubMed]

36. Taechoyotin, P.; Tongrod, P.; Thaweerungruangkul, T.; Towattananon, N.; Teekapakvisit, P.; Aksornpusitpong, C.; Sathapornpunya, W.; Hempatawee, N.; Rangsin, R.; Mungthin, M. Prevalence and associated factors of internet gaming disorder among secondary school students in rural community, Thailand: A cross-sectional study. BMC Res. Notes 2020, 13, 1-7. [CrossRef] [PubMed]

37. Clement, J. Global Internet Penetration Rate as of January 2020, by Region. Available online: https: //www.statista.com/statistics/269329/penetration-rate-of-the-internet-by-region/ (accessed on 9 March 2020).

38. Cheng, C.; Li, A.Y.L. Internet Addiction Prevalence and Quality of (Real) Life: A Meta-Analysis of 31 Nations Across Seven World Regions. Cyberpsychol. Behav. Soc. Netw. 2014, 17, 755-760. [CrossRef]

39. Müller, K.W.; Janikian, M.; Dreier, M.; Wölfling, K.; Beutel, M.; Tzavara, C.; Richardson, C.; Tsitsika, A. Regular gaming behavior and internet gaming disorder in European adolescents: Results from a cross-national representative survey of prevalence, predictors, and psychopathological correlates. Eur. Child Adolesc. Psychiatry 2015, 24, 565-574. [CrossRef]

40. Gentile, D. Pathological video-game use among youth ages 8 to 18: A national study. Psychol. Sci. 2009, 20, 594-602. [CrossRef]

41. Oyserman, D.; Lee, S.W.S. Does Culture Influence What and How We Think? Effects of Priming Individualism and Collectivism. Psychol. Bull. 2008, 134, 311-342. [CrossRef]

42. Oyserman, D.; Coon, H.M.; Kemmelmeier, M. Rethinking Individualism and Collectivism: Evaluation of Theoretical Assumptions and Meta-Analyses. Psychol. Bull. 2002, 128, 3-72. [CrossRef]

43. Arpaci, I.; Kesici, S.; Baloğlu, M. Individualism and internet addiction: The mediating role of psychological needs. Internet Res. 2018, 28, 293-314. [CrossRef]

44. Li, M.; Xue, H.; Wang, W.; Wang, Y. Parental expectations and child screen and academic sedentary behaviors in China. Am. J. Prev. Med. 2017, 52, 680-689. [CrossRef]

45. Goh, W.W.; Bay, S.; Chen, V.H.-H. Young school children's use of digital devices and parental rules. Telemat. Inform. 2015, 32, 787-795. [CrossRef]

46. Erikson, E. Psychosocial Development; Norton: New York, NY, USA, 1982.

47. Kim, Y.-Y.; Kim, M.-H. The impact of social factors on excessive online game usage, moderated by online self-identity. Clust. Comput. 2017, 20, 569-582. [CrossRef]

48. Šporčić, B.; Glavak-Tkalić, R. The relationship between online gaming motivation, self-concept clarity and tendency toward problematic gaming. Cyberpsychol. J. Psychosoc. Res. Cyberspace 2018, 12. [CrossRef] 
49. Park, S.; Jeon, H.J.; Bae, J.N.; Seong, S.J.; Hong, J.P. Prevalence and psychiatric comorbidities of Internet addiction in a nationwide sample of Korean adults. Psychiatry Investig. 2017, 14, 879. [CrossRef] [PubMed]

50. Ho, R.C.; Zhang, M.W.; Tsang, T.Y.; Toh, A.H.; Pan, F.; Lu, Y.; Cheng, C.; Yip, P.S.; Lam, L.T.; Lai, C.-M. The association between internet addiction and psychiatric co-morbidity: A meta-analysis. BMC Psychiatry 2014, 14, 183. [CrossRef] [PubMed]

(C) 2020 by the authors. Licensee MDPI, Basel, Switzerland. This article is an open access article distributed under the terms and conditions of the Creative Commons Attribution (CC BY) license (http://creativecommons.org/licenses/by/4.0/). 